\title{
Optoelectronic Sensor Solid Surface Color Analyzer
}

\author{
Radjabov Telman, Rakhimov Bakhtiyor, Fayzullayev Nasrulla \\ Tashkent University of Information Technologies Named After Muhammad al-Khwarizmi, Faculty of Telecommunications Technologies, \\ Tashkent, Uzbekistan
}

Email address:

b.rahimov@tuit.uz(R. Bakhtiyor),brah2008@rambler.ru(R. Bakhtiyor)

\section{To cite this article:}

Radjabov Telman, Rakhimov Bakhtiyor, Fayzullayev Nasrulla. Optoelectronic Sensor Solid Surface Color Analyzer. Journal of Biomaterials. Vol. 2, No. 1, 2018, pp. 12-15. doi: 10.11648/j.jb.20180201.13

Received: March 14, 2018; Accepted: April 20, 2018; Published: May 21, 2018

\begin{abstract}
In the paper, studies of fiber transmission lines have been considered, for measurements of distances in space and atmosphere, the study of atoms and molecules and in solving other problems of science and technology. The article deals with one of the pulse methods of fiber optics, i.e. scatterometry or backscattering for metal surfaces qualitative parameters control. This object can be apply not only to radio electronics, but also to physics, chemistry, biology and other areas of science and technology.
\end{abstract}

Keywords: Analyzer, Optical Fibers, RGB-System, Light-Emitting Diode

\section{Introduction}

At present, optoelectronic methods of control are successfully used for quantitative and qualitative analysis of various substances, for example, for determining the parameters of liquid semitransparent media (oil products, vegetable oil, glycerin, juices, drinks, urine, blood, etc.). Compared to other physicochemical methods of analysis, they have significant advantages such as high accuracy, sensitivity and economy. The essence of optoelectronic monitoring is that any substance reflects, absorbs, or emits light. Depending on the chemical composition of the substance and the quantitative ratio of its constituent elements, the intensity of light, the absorption coefficient, the reflection angle, and other characteristics of the interaction of light radiation and matter change[1-2].

Color sensor systems are increasingly being used in automated applications to detect automation errors and monitor quality at the speed of production line. They are used in assembly lines to identify and classify products by color. The objectives of their usage include to check the quality of products [3-5], to facilitate sorting and packaging [6-8], to assess the equality of products in storage $[9,10]$, and to monitor waste products [11]. Consequently, there is abundant of color sensors and the choice is often application-driven [8, 16]. Low cost and simple color sensors are preferred over sophisticated solutions for less demanding applications where the top priority is cost and power consumption. This paper presents such a sensor that is based on an embedded analogto-digital converter (ADC) as the end-user interface circuit. The proposed system is capable of differentiating up to eight different colors and thus, it is specifically targeted for automated applications where few colored products need to be detected.

\section{Analyzer of Solid Materials}

Further in this chapter the optoelectronic automatic analyzer for measurement of color parameters of the surface of solid materials, for example, metals, plastic, glass, paper, etc., in those areas of the industry where color is one of the main indicators of quality of production is described.

Filter colorimeters containing light sources, correcting light filters $(\mathrm{X}),(\mathrm{Y}),(\mathrm{Z})$ and a photocell are known.

However, this device controls on the principle of passage, analyzes the colors of only translucent fluids, and also has a complex design.

The surface color analyzer of solid materials consists of an electronic unit containing a master oscillator and a trigger switch, a sensor containing three pairs of measuring LEDs and three pairs of compensating light emitting diodes, three measuring instruments, and a measuring system. According to the invention, three triggers connected to the trigger switch are connected to it, three identical optical radiation receivers are located behind the sensor in the signal path, three comparison units that receive signals from the respective 
optical radiation receivers, a photoelectric signal processing unit that is connected to the three comparison units and a memory associated with the processing unit, in addition, the sensor is in the form of a hemisphere, with an attached annular casing of soft rubber to which is attached Three pairs of optical fibers disposed at an angle, for example $45^{\circ}$, relative to each other and symmetrically with respect to the normal to the test surface at the reflection point.
In figure 1 is a block diagram of the device for analyzing the color of the surface of solid materials, and in figure 2 one of the embodiments of the sensor. The color analyzer consists of a sensor and an electronic unit. The sensor is made in the form of a hemisphere 1, in which are installed three pairs of Y-shaped supply 2-4 and withdrawing 5-7 optical fibers.



Figure 1. Structural schematic of color analyzer of the solid surface.
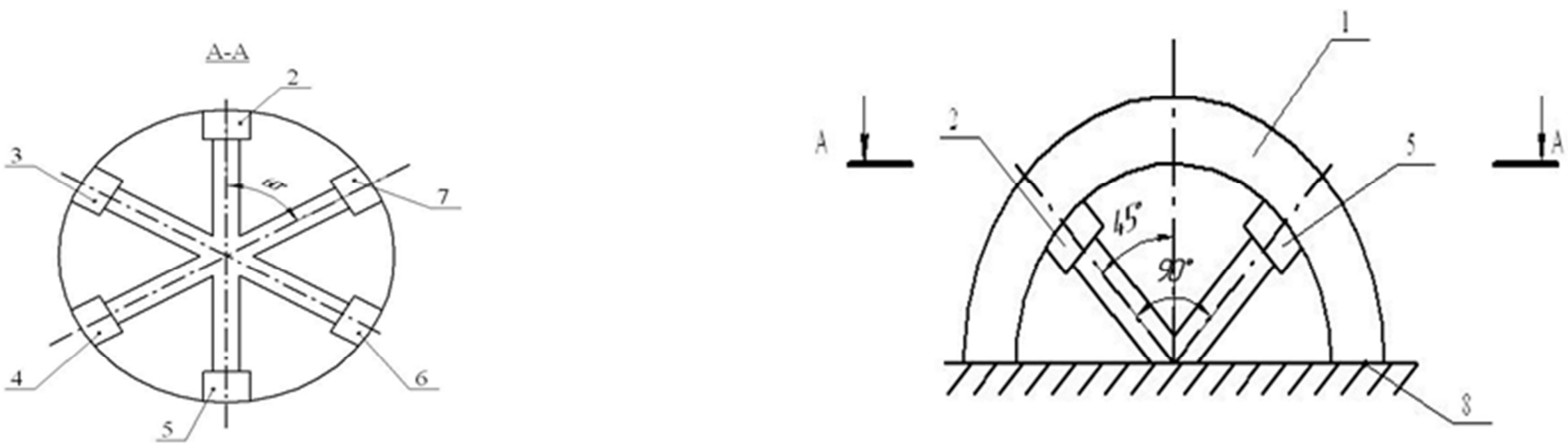

Figure 2. One embodiment of the sensor.

Narrow beam radiation, which allows control of parameters is beeped and received due to the flow of the light through the leading 2-4 and offtake 5-7 fiber optic.

The device of the analyzer of the surface color of solid materials in figure 2, and in figure 1 - one of options of sensor performance. The analyzer of color consists of the sensor and the electronic block. The sensor is executed in the form of a hemisphere, 1 in which three couples of $\mathrm{Y}$ figurative bringing 2-4 and taking away 5-7 optical fibers are established.

Physical meaning consists of the following: color parameters are detected as an objective features of the subject developing in spectral composition outcoming from them (transmitted and attracted) radiation and perceived as visual sensation.

\section{The Device Works as Follows}

The master oscillator 9 generates pulses that are supplied to the input of the switch 10 . The separating pulses are fed to the input of three identical flip-flops 11-13, three outputs of which are connected to three measuring LEDs $14,16,18$, the second three outputs are equipped with compensation LEDs $15,17,19$ pulses from the triggers are fed to the corresponding LEDs. Each optocoupler is responsible for monitoring a particular parameter.

The monitored surface 8 , which is enclosed in the hemisphere 1, is irradiated by two optical fluxes (measuring and compensating) through the supply optical fibers 2 - 4 .

Optoelectronic pairs are enclosed in a soft rubber casing for the necessary orientation of the sensor and optical isolation of the optical channel and are located at an angle of, for example, $45^{\circ}$, relative to each other and symmetrically with respect to the normal to the monitored surface at the reflection point.

The optical radiation is reflected from the monitored surface and the outgoing optical fibers 5-7 are fed to optical radiation receivers 20-22 operating at wavelengths $\lambda_{1}=680$ 
$\mathrm{nm} \lambda_{2}=560 \mathrm{~nm} \lambda_{3}=450 \mathrm{~nm}$ and converting the optical signals into electrical ones. Due to the passage of light through the supply and discharge optical fiber, a narrow beam of radiation is fed and received, which makes it possible to control the parameters.

Further, the signal falls on its comparison unit 23, 24, 25, the ratio of the two signals (measuring and compensating) is taken and further the measuring system 26, 27, 28 determines shades of three colors. The measurement process at this stage can be completed. Or, three signals can be fed to the photoelectric signal processing unit 29, where they are matched to any of a series of exemplary stored in the memory 30 . Then both signals or their ratio are applied to the measurement system or to the computer 31 .

The physical meaning is as follows: color parameters are defined as an objective property of objects, which is manifested in the spectral composition of the radiation emitted from them (transmitted, reflected) and perceived as a conscious visual sensation. In this definition, two aspects are given - physical and psycho-physiological, inextricably

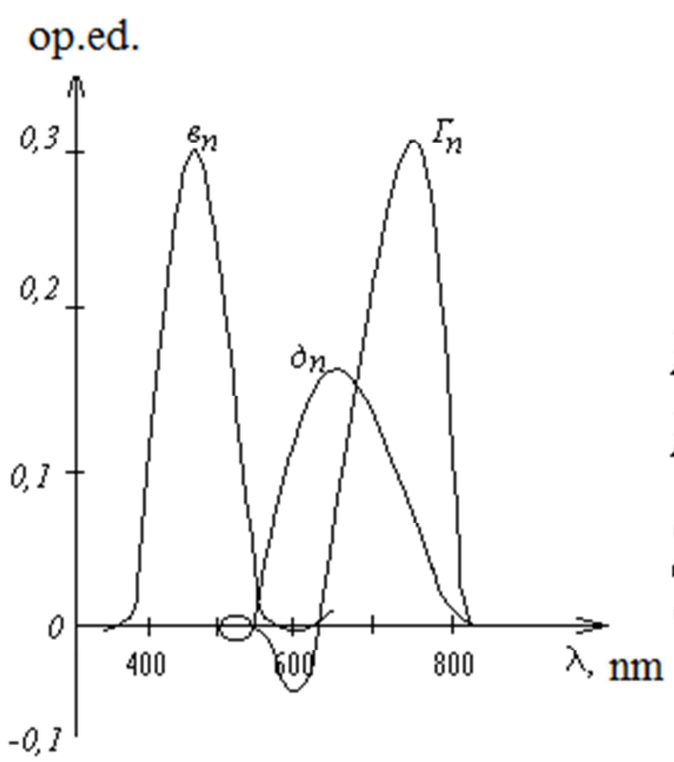

linked to each other.

The modern theory of color recognition is based on the uniquely established fact of human eye trichromatism, i.e. the visual apparatus contains three types of receptors, each of which predominantly reacts to red, green or blue.

\section{Characterization}

According to this, the color parameter is mathematically expressed by a vector in three-dimensional color space, and the beginning of this vector coincides with the beginning of the color coordinate system (CCS). If we use the unit vectors of three colors of red rn, green gn and blue bn as the primary colors, then any color can be expressed as:

$$
\mathrm{Y}=\mathrm{R}_{\mathrm{r}_{\mathrm{n}}}+G_{g_{n}}+\mathrm{B}_{b_{n}}
$$

where R, G, B are the qualities of the corresponding colors.

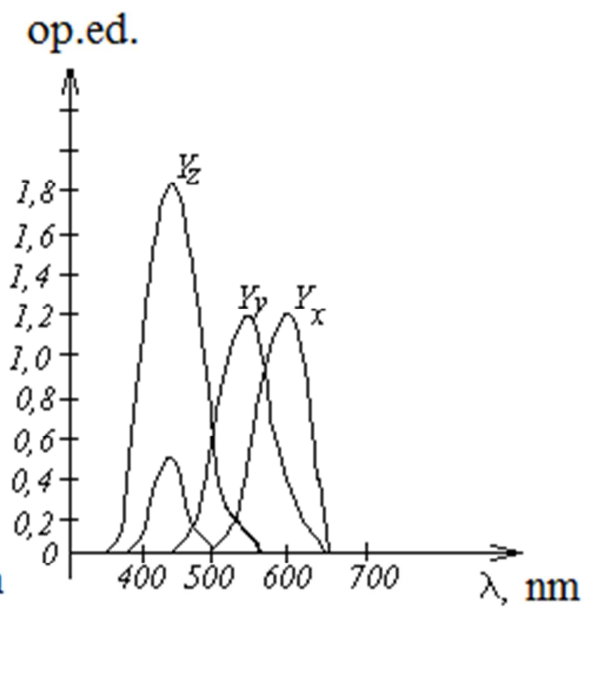

Figure 3. Characteristics of sensors a color analyzer of the surface of solid materials.

In figure 3. the curves for the addition of colors of the systems RGB (a) and XYZ (b) are shown. RGB-system is empirical, which is used as the primary colors of pure spectral radiation of red $(\lambda=700 \mathrm{~nm})$, green $(\lambda=546.1 \mathrm{~nm})$ and blue $(\lambda=435.8 \mathrm{~nm})$ colors. And $\mathrm{XYZ}$ is a phenomenological system. The meaning of these systems is that the spectrally pure color with $\lambda=600 \mathrm{~nm}$ is perceived by the eye as consisting of a red and green component in a 14: 3 ratio, radiation with $\lambda=450 \mathrm{~nm}$ is perceived as a ratio of 7.5: 1: 35 red, green and blue colors respectively.

In the RGB system, the curves of red $r_{n}$, green $g_{n}$ and blue $b_{n}$ are constructed so that for each of the three primary colors only the ordinate of one curve differs from zero. The main disadvantage of these addition curves is the presence of a negative section near the curve. When measuring color coordinates, color subtraction cannot be realized, therefore, in colorimetric this system is not applied.

Thus, the task of controlling color parameters based on the $\mathrm{XYZ}$ system is carried out in three ways. The first is a visual comparison of the measured color with the reference one. The standard is chosen from a pre-compiled color atlas or by computer programming of each of the colors. The second is the spectrophotometry of the observed radiation and the calculation of the $\mathrm{X}, \mathrm{Y}, \mathrm{Z}$ coordinates. The third is the direct measurement of the $\mathrm{X}, \mathrm{Y}, \mathrm{Z}$ coordinates using three reference optical radiation receivers, the spectral functions whose sensitivities exactly correspond to the $\mathrm{Y}_{\mathrm{x}} \mathrm{Y}_{\mathrm{y}} \mathrm{Y}_{\mathrm{z}}$ curves. This method is undoubtedly the most promising, since it meets the requirements of the technological process.

To develop this method, the device must perform the following operations:

a. the isolation of the analyzing radiation at three 
wavelengths (red, green and blue);

b. receiving the radiation fluxes reflected from the monitored object and converting them into a photoelectric signal;

c. -processing and comparison of the photoelectric signal.

The purpose of each of the three optocouplers is explained as follows: the monitored surface is irradiated with two light streams with wavelengths $\lambda_{1}$ and $\lambda_{2}$, one of which is measurement radiation, and the other is compensatory radiation.

Let the light flux $\mathrm{f}_{0}$ падает fall on the monitored surface. The illuminated layer will divide the light flux incident onto it into three parts:

1) $f_{\lambda 1}$ - reflected from the surface and incident on the receiver of optical radiation, from which came the falling stream;

2) $f_{\lambda_{2}}$ - reflected from the surface and not incident on the receiver of optical radiation;

3) $f_{\lambda 3}$ - absorbed stream, which in the substance of the layer will turn into heat or another form of energy.

In accordance with the law of conservation of energy, the sum of the light fluxes is equal to the incident flux:

$$
\mathrm{f}_{01}=\mathrm{f}_{\lambda 1}+\mathrm{f}_{\lambda 2}+\mathrm{f}_{\lambda 3}
$$

Or

$$
\frac{\mathrm{f}_{\lambda 1}}{\mathrm{f}_{01}}+\frac{\mathrm{f}_{\lambda 2}}{\mathrm{f}_{0 \lambda}}+\frac{\mathrm{f}_{\lambda 3}}{\mathrm{f}_{0 \lambda}}=1
$$

For colored substances, these coefficients depend on the spectral composition of the incident radiation. For monochromatic radiation with a certain wavelength (as the emission spectrum of a light-emitting diode), we denote $\rho$ $(\lambda), \tau(\lambda)$, and $\alpha(\lambda)$.

$$
\lambda \mp \frac{1}{2} \delta \lambda
$$

The spectral dependence of these coefficients is conveniently depicted graphically.

\section{Conclusions and Outlook}

The proposed device has improved color recognition accuracy due to three opt couplers that control three color parameters corresponding to the parameters $\mathrm{X}, \mathrm{Y}, \mathrm{Z}$.

The device has increased accuracy of control due to threedimensional measurement with wavelengths $\lambda_{1}=680 \mathrm{~nm}$, $\lambda_{2}=560 \mathrm{~nm}, \lambda_{3}=450 \mathrm{~nm}$, when multi-color photo resistors are used as standard optical radiation receivers.

If necessary, the signal from the output of the photoelectric signal processing unit can be fed into the automatic control system.

\section{References}

[1] Rakhimov B. N, Ushakov O. K, Larina T. V, Kutenkova E. $\mathrm{Yu}$. The analyzer of color of a surface of firm materials//the Scientific and technical magazine "Devices and the technician of experiment" -Moscow, 2012, №3- p. 131-132.

[2] Pat. № 2429456 of the Russian Federation. Analyzer of the color of the surface of solid materials. / Rakhimov B. N, Kutenkova E. Yu., Larina T.V, Ushakov O.K / / 20.09.2011. Bul. №26,

[3] C. Zhang and K. S. Suslick, "Colorimetric sensor array for soft drink analysis," J. Agric. Food Chem., vol. 55, pp. 237242, 2007.

[4] P. D. Oram and J. Strine, "Color measurement of a solid active pharmaceutical ingredient as an aid to identifying key process parameters," J. Pharm. Biomed. Anal., vol. 40, pp. 1021-1024, 2006.

[5] M. Frank, et al., "High-speed industrial color and position sensors," Electronic Imaging'99, pp. 50-57, 1999.

[6] A. Pacquit, et al., "Development of a smart packaging for the monitoring of fish spoilage," Food Chem., vol. 102, pp. 466470, 2007.

[7] E. J. Cadieux Jr, et al., "System and method for visually inspecting a cigarette packaging process," ed: Google Patents, 2002.

[8] D.-J. Lee and R. S. Anbalagan, "High-speed automated colorsorting vision system," in Optical Engineering Midwest'95, pp. 573-579, 1995.

[9] J. V. Popov-Raljić, et al., "Investigations of bread production with postponed staling applying instrumental measurements of bread crumb color," Sensors, vol. 9, pp. 8613- 8623, 2009.

[10] J. V. Popov-Raljić and J. G. Laličić-Petronijević, "Sensory properties and color measurements of dietary chocolates with different compositions during storage for up to 360 days," Sensors, vol. 9, pp. 1996-2016, 2009.

[11] R. Bogue, "Optical chemical sensors for industrial applications," Sensor Review., vol. 27, pp. 86-90, 2007.

[12] R. Baribeau, et al., "Color reflectance modeling using a polychromatic laser range sensor," IEEE T Pattern. Anal., vol. 14, pp. 263-269, 1992.

[13] B. V. Zeghbroeck, "p-n Junctions," Principles of Semiconductor Devices, ed: http://ecee.colorado.edu/ bart/book/, 2011.

[14] M. J. Deen and P. K. Basu, "Silicon Photodetectors," Silicon Photonics, ed: John Wiley \& Sons, Ltd, 2012, pp. 197-247.

[15] H. L. L. Ling, "Integrated Photodiode and Amplifier OPT301," International Electronic Elements, vol. 12, p. 017, 2002.

[16] G. Torfs, et al., "Low-power 4-bit flash analogue to digital converter for ranging applications," Electronics letters, vol. 47, pp. 20-22, 2011.

[17] Rakhimov B. N, Abdukhalilov B. Locate objects mechanical damage based on fiber-optic communication// Asian Journal of Research № 6(6), Japan, Osaka, July 2017, p. 117-126. 